\title{
Kirchen auf neuen Fernseh-Kanälen?
}

\author{
von Konrad W. Kraemer
}

Schon im Jahre 1971 wird das Fernsehen in der Bundesrepublik eine Ausweitung erfahren. Noch steht nicht fest, ob es ein bundesweit ausgestrahltes Viertes Fernsehprogramm oder eine Vielzahl von Regional- oder sogar Lokalprogrammen geben wird. Der nach mehrjährigen Forschungs- und Entwicklungsarbeiten neu erschlossene Frequenzbereich (12 Giga-Hertz/Band VI) bietet die technischen Voraussetzungen für beides, natürlich auch für eine Kombination. Entsprechende Ankündigungen des Bundesministers für das Post- und Fernmeldewesen, Dr. Werner Dollinger, haben die Diskussion über Struktur, Trägerschaft und Programmkonzeption eines weiteren Fernsehprogramms schlagartig belebt.

\section{Die fernsebtechnische Entwicklung}

Zunächst ein Wort zu den technischen Aspekten. Die in Stockholm 1952 und 1961 dem Rundfunk zugeteilten Frequenzbereiche ergaben für das Fernsehen die Bänder I und III bis V mit einer Bandbreite von insgesamt $400 \mathrm{Mega-Hertz}$, die jedoch nur die drei jetzt bestehenden Programme zulassen. Bereits im Jahre 1959 hatte man in Genf auf deutschen Antrag das Band VI - den sogenannten 12-Giga-HertzBereich - für künftige Entwicklungen in Aussicht genommen. Die Frequenzen dieses Bereichs liegen zwischen 11,7 und 12,7 Giga-Hertz, die verwendeten Wellenlängen bewegen sich damit zwischen 2,5 und $2,7 \mathrm{~cm}$.

$\mathrm{Da}$ ein Fernsehen in diesem Bereich am besten in Ballungsgebieten wirtschaftlich arbeiten kann, entsteht zur Zeit in Berlin das erste deutsche Versuchsnetz der Deutschen Bundespost. Neben diesem für wissenschaftliche Untersuchungen gedachten Versuchsnetz wird die Deutsche Bundespost etwa Mitte 1971 ein von der deutschen Industrie entwickeltes Erprobungsnetz in Betrieb nehmen, das bis zu zehn Sender und etwa 200 Empfangskonverter umfassen wird. Für den Erfolg der Bemühungen, ein preislich günstiges Empfangsgerät zu schaffen, waren übrigens die Erkenntnisse aus der Entwicklung der Nachrichtengeräte für Satelliten und Raumfahrt bestimmend. Es konnte ein röhrenloser Empfangskonverter geschaffen werden, der zumindest für Gemeinschaftsantennen-Anlage wirtschaftlich vertretbar ist. Nächstes Ziel ist die Entwicklung eines Konverters, der auch für Fernsehteilnehmer ohne Gemeinschaftsantenne erschwinglich ist.

Im 12-Giga-Hertz-Bereich wird der Empfangskonverter voraussichtlich zur Dauereinrichtung werden. In diesem Bereich sind die Verluste auf den Zuführungskabeln wesentlich größer als in den bisher gebräuchlichen Bändern. Es erscheint deshalb

Dr. Konrad W. Kraemer MA ist Chefredakteur der Katholischen Nachrichtenagentur (KNA; Bonn, München, Berlin, Hamburg, Frankfurt, Rom). Das Thema des für CS geschriebenen Originalbeitrags war auch Gegenstand eines Referates, das der Verfasser beim 14. Evangelisch-katholischen Publizistentreffen (2. bis 4. Mai 1969) in Maria Laach hielt. 
zweckmäßig, möglichst dicht hinter der Antenne auf eine niedrigere Frequenz umzusetzen. Mit dieser Technik ist es möglich, die bisherigen Fernsehempfänger beizubehalten.

Für den 12-Giga-Hertz-Bereich sind besondere Empfangsantennen erforderlich. Die kurze Wellenlänge erlaubt die Verwendung einer kleinen Antenne. Dadurch wird die Verwendung von Parabolspiegeln mit einem maximalen Durchmesser von 60 Zentimetern möglich, die sich für eine Massenfertigung eignen. Die hohe Richtwirkung dieser Parabolantenne ermöglicht es, mit kleinen Sendeleistungen auszukommen. Mehr noch als im Bereich V, der für die Ausstrahlung des II. und III. Fernsehprogrammes verwendet wird, ist im Bereich VI direkte Sichtverbindung zwischen Sende- und Empfangsantenne erforderlich. Es gibt also "viel Schatten" im 12-GigaHertz-Bereich. Der Versorgungsradius eines Senders wird daher wenig größer als 20 Kilometer sein.

\section{Rechtliche Grundlagen}

Im Hinblick auf die langfristige Entwicklung des Fernsehens, die ähnlich wie in den USA und in Japan auch bei uns fast zwangsläufig zu einer größeren Zahl von Programmen führen wird, kann auch die Diskussion darüber nicht als beendet gelten, ob die öffentlich-rechtliche Struktur des Rundfunks prinzipiell Grundlage der Organisation von Hörfunk und Fernsehen in der Bundesrepublik bleiben soll. Sie hat sich darum nach etwa acht Jahren Unterbrechung erneut einer Erörterung der Möglichkeiten zur Schaffung eines privaten Fernsehens zugewandt.

Alle Uberlegungen in dieser Richtung setzen eine Rückblende auf das richtungweisende Fernsehurteil des Bundesverfassungsgerichtes vom 28. Februar 1961 voraus. Damals war die Gründung der „Deutschland-Fernsehen-GmbH“ durch den Bund, der auch die für die Bundesländer vorgesehenen Anteile treuhänderisch verwaltete, für grundgesetzwidrig erklärt worden. Keineswegs - diese Klarstellung ist für die neuerlichen Erörterungen wesentlich - hat das Bundesverfassungsgericht seinerzeit ein privates Fernsehen inhibiert. Es hat im Gegenteil ausdrücklich festgestellt, daß auch eine rechtsfähige Gesellschaft des privaten Rechts Träger von Fernsehveranstaltungen sein kann, und dafür als Bedingung die hinreichende Gewähr genannt, daß „alle gesellschaftlich relevanten Kräfte zu Wort kommen und die Freiheit der Berichterstattung unangetastet bleibt".

Es darf deshalb davon ausgegangen werden, daß - neben den technischen - auch die rechtlichen Voraussetzungen für ein privates Fernsehen geschaffen werden können. Die Auflagen der Verfassungsrichter führen nun direkt zu der Frage der Trägerschaft eines Vierten Fernsehprogramms. Die CDU hat dazu auf ihrem Bundesparteitag in Berlin folgende Formulierung in ihr Aktionsprogramm aufgenommen: „Die Ausstrahlung weiterer Fernsehprogramme durch andere Veranstalter soll unter öffentlicher Aufsicht und unter Beteiligung aller gesellschaftlich relevanten Kräfte möglich sein. " Uneingeschränkt zu bejahen ist die Forderung nach öffentlicher Kontrolle, für die es bei ARD und ZDF bewährte Formen und Modelle gibt. Einer genaueren Interpretation bedarf hingegen der Begriff „Beteiligung“. Ist damit Beteiligung an der Trägerschaft oder an der Aufsichtsfunktion gemeint? Das Bundesverfassungsgericht postulierte 1961, „daß alle gesellschaftlich relevanten Kräfte zu Wort kommen". Diese Formulierung zielt eher auf die wirksame Wahrnehmung der Kontrollfunktion als auf eine Beteiligung an der Trägerschaft ab. 


\section{Struktur und Trägerschaft}

An einer Trägerschaft ist in erster Linie die Presse - vertreten durch die Pressevereinigung für neue Publikationsmittel e. V. - interessiert, die seit Jahren den durch das konkurrierende Werbefernsehen bedingten Anzeigenausfall beklagt und hier nach einem langfristigen Ausgleich sucht. Zurzeit bestehen innerhalb der Presse noch stark divergierende Ansichten über die Art und Weise einer Beteiligung. Doch ist unschwer die Prognose zu stellen, daß ein Viertes Programm nicht ausschließlich der Presse oder gar nur bestimmten Pressegruppen anvertraut werden wird. Die Eröffnung einer Beteiligungsmöglichkeit für alle Zeitungs- und Zeitschriftenverlage, die eine solche Beteiligung wünschen, kann hingegen nur begrüßt werden.

Als weitere Träger dürften die Parteien, die Kirchen sowie die Organisationen der Sozialpartner infrage kommen. Bekundungen eines grundsätzlichen Interesses liegen vor und haben sich im Saarland nach der Schaffung der gesetzlichen Grundlage für die Konzessionierung einer kommerziellen Anstalt bereits konkret manifestiert. Beachtenswert ist ferner, daß die SPD in ihrem Pressedienst wohlwollend zu einer "Aktualisierung" des Fernsehurteils von 1961 Stellung nahm und eine baldige Anpassung der Rechtslage an die technische Fortentwicklung im Fernsehbereich befürwortete. Auch die FDP - von jeher ein Freund eines privaten Fernsehens hat durch die Vorlage des Entwurfs eines neuen Rundfunkgesetzes für Berlin im Januar $1969 \mathrm{zu}$ erkennen gegeben, daß sie unverändert die Zulassung privater Fernsehveranstalter anstrebt.

\section{Die Haltung der Kirchen}

In den Kirchen ist die Diskussion noch im Gange. Einen bemerkenswerten Beitrag leistete die Kammer für publizistische Arbeit der Evangelischen Kirche in Deutschland mit ihrem Sachverständigengutachten zum Thema "Gesellschaft und öffentliche Kommunikation" vom 15. November 1968. Das Gutachten äußert sich positiv zu einer privatwirtschaftlichen Organisation weiterer Fernsehprogramme und geht dabei insofern über das Fernsehurteil von 1961 hinaus, als es Sendelizenzen für einzelne leistungsfähige Veranstalter für möglich hält. In diesem Zusammenhang wird ausdrücklich auf eine Beteiligung der Kirchen wie auch der Universitäten und Hochschulen hingewiesen.

Aus den zahlreichen interessanten Gedanken der Gutachter sei die Möglichkeit herausgegriffen, daß internationale Sendegesellschaften ihre Programme über Satelliten an Sender bzw. Sendenetze im Zentimeter-Band (Giga-Hertz-Bereich) übermitteln könnten. Schließlich ist es keine Utopie, sondern lediglich eine andere Applizierung des gleichen Prinzips, nicht nur an den Westen, sondern auch an den Osten, und nicht nur an Unterhaltungs-, sondern auch an politische Propagandasendungen zu denken.

Hinsichtlich des geltenden Verfassungsrechtes merken die Gutachter der EKD an, daß das Sendemonopol der durch Staatsverträge errichteten Rundfunkanstalten stets mit der geringen Zahl der zur Verfügung stehenden Frequenzen begründet wurde. Darum habe ihr Programm alle gesellschaftlich relevanten Kräfte berücksichtigen müssen. Die neue Entwicklung der Rundfunktechnik spricht demnach für eine Aufhebung des noch bestehenden Monopols der öffentlich-rechtlichen Anstalten. 
Die Gutachter ordnen dieses Moment bei einem Ausblick auf die Möglichkeit einer schnellen Veränderung des Verhältnisses von Presse und Rundfunk ein. Dieser Gedanke wurde wiederum sehr bald von der Pressevereinigung für neue Publikationsmittel aufgenommen. Fußend auf den Ergebnissen einer ständigen Beobachtung der Wirkung des Fernsehens als Informationsquelle über das Welt- und Tagesgeschehen durch das Bielefelder EMNID-Institut (Mitgliederrundschreiben Nr. 220 vom 11. 2. 1969) präsentierte sie überraschende Ziffern zu den „Gefahren eines Informationsmonopols auf Grund der geringen Anzahl der Fernsehprogramme“. Im Interesse eines vielfältigen Informationsangebotes wird auch hier für eine Vermehrung der Fernsehprogramine - unter Beteiligung der Zeitungsverleger - plädiert.

Seitens der katholischen Kirche liegt bislang noch keine offizielle Stellungnahme vor. Doch ist bekannt, daß sich jetzt weitgehend eine Bereitschaft zur konstruktiven Mitwirkung auch an einem privaten Fernsehen abzeichnet. Zwar würde auch heute für die katholische Kirche ein "rein kommerzielles" Fernsehen gewiß ein Stein des Anstoßes sein. Es ist jedoch festzuhalten, daß eine Gesellschaft privaten Rechts als Veranstalter von Fernsehsendungen keineswegs mit einem kommerziellen, also ausschließlich auf Gewinnstreben ausgerichteten Fernsehen, identisch sein muß.

Insbesondere unter Berücksichtigung der wohlverstandenen eigenen Interessen und ihrer Aufgabenstellung in der modernen Welt hat sich die Haltung der katholischen Kirche zu Rundfunk und Fernsehen in den vergangenen Jahren weiterentwickelt. Die betont defensive Einstellung der Enzyklika "Miranda prorsus" vom 8. September 1957 findet sich im Konzilsdekret „Inter mirifica" nicht mehr. Dort heißt es, daß der Kirche das ursprüngliche Recht zusteht, alle Werkzeuge der sozialen Kommunikation nicht nur $\mathrm{zu}$ benutzen, sondern auch $\mathrm{zu}$ besitzen. In Deutschland aber ließe sich dieses ursprüngliche Recht der Kirche nicht im Rahmen öffentlich-rechtlicher Strukturen, sondern allenfalls durch eine Beteiligung in privatrechtlichem Rahmen verwirklichen.

Ein wesentliches Argument kirchlicher Persönlichkeiten gegen ein privates Fernsehen war die Sorge, daß im Wettbewerb verschiedener programmgestaltender Kräfte die von kommerziellen Gesichtspunkten ausgehenden die stärksten sein und in Anpassung an das Erwartungsniveau des breiten Publikums einen "Zug nach unten" bewirken würden. Abgesehen davon, daß diese Auffassung unter verschiedenen Aspekten fragwürdig war, scheint sie auch durch die technische Entwicklung praktisch überholt. Mehr und mehr wird die individuelle Programmgestaltung eine Frage der Auswahl aus einem gefächerten Angebot.

In nicht allzu ferner Zeit wird die Programmplanung und -gestaltung durch die neuen Techniken der Konservierung von Fernsehsendungen völlig der interessenund geschmacksbestimmten Entscheidung der Rezipienten unterliegen. Der heute schon bekannte Heim-Recorder zum Mitschneiden von Sendungen stellt nur eine primitive Vorstufe dar. Endpunkt der Entwicklung wird ein zentrales Fernseharchiv sein, in dem sämtliche Sendungen von bestimmtem Wiederholungswert gespeichert und jederzeit für jedermann auf Abruf überspielt werden können. Ergänzend sei darauf hingewiesen, daß auch das Problem der Textübermittlung und -kopierung technisch gelöst ist (Zeitung, Lehrbuch für den audiovisuellen Unterricht, etc.).

Dies sind keine Utopien, sondern mit prognostischen Methoden erarbeitete Ergebnisse der wissenschaftlichen Futurologie (Planungswissenschaft). Alexander von Cube zog daraus unlängst in einem Vortrag das Fazit: „Der Programm-Macher der Zukunft ist das Publikum." Die Kirchen haben aus dieser zweifelsohne richtigen Erkenntnis m. E. eine doppelte Aufgabe zu deduzieren: intensivierte Medienerzie- 
hung und -bildung sowie aktive Förderung eines möglichst qualifizierten Programmangebots. Eine reale Chance bietet neben der vollen Ausschöpfung der bei den öffentlich-rechtlichen Anstalten gebotenen Möglichkeiten die Beteiligung an einem privatrechtlich strukturierten Sender.

$\mathrm{Da}$ ein leistungsfähiger Sender einer soliden und möglichst nicht allzu heterogenen Trägerschaft bedarf, bietet sich vielleicht eine Partnerschaft $\mathrm{z}$ wischen den Kirchen, der Presse und einer Gemeinschaft kultureller Institutionen als praktikable Lösung an. Im Bereich der Presse sollte dann auch die kirchliche Presse angemessen vertreten sein. Diese vier „tragfähigen Säulen“ wurden übrigens bereits 1964 auf dem "Kleinen Katholikentag“ in Münster von dem Bundesverfassungsrichter Prof. Dr. Willi Geiger in Vorschlag gebracht.

\section{Probleme der Finanzierung}

Großes Gewicht werden natürlich die Fragen der Finanzierung haben. Bekanntlich liegen die Investitionskosten für eine neue Fernsehanstalt bei ca. 120 bis 150 Millionen D-Mark. Für weitere Jahre muß mit jeweils 50 bis 60 Millionen D-Mark an Zuschüssen gerechnet werden. Die in Großbritannien, Kanada und USA gemachten Erfahrungen zeigen, daß erst nach etwa sechs bis zehn Jahren mit einem ausgeglichenen Haushalt beziehungsweise Gewinnen gerechnet werden kann. Andererseits gilt es, das unzutreffende Argument auszuräumen, daß das gesamte Investitionskapital von den Gesellschaftern der Trägergesellschaft aufgebracht werden müßte.

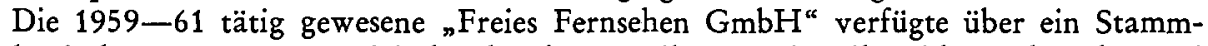
kapital von 200000 D-Mark, das in Anteile von jeweils nicht mehr als zwei Prozent aufgestückelt wurde. Es erscheint keineswegs als unmöglich, die zweifellos hohen Investitionskosten auf dem Kapitalmarkt aufzubringen, da die Aussicht auf Amortisation in absehbarer Zeit und künftige Gewinne einen sicheren Grad von Gewißheit hat.

\section{Lokalprogramme keine Lösung}

Schließlich ist der Fragenbereich von Programmstruktur und Programmproduktion $\mathrm{zu}$ prüfen, der in engem Zusammenhang mit den technischen Bedingungen des neu erschlossenen Frequenzbereiches steht. Es sind Empfangskonverter und besondere Empfangsantennen erforderlich. Der Versorgungsradius eines Senders wird kaum größer als 20 Kilometer sein. Die einzelnen Sender können jedoch zu einem überregionalen Sendernetz zusammengefaßt werden. Viel zu wenig beachtet wurde übrigens bisher die Möglichkeit der Kombination der neuen Fernsehanstalt mit dem Hörfunk. Die "Entwicklungsstufe" eines privaten Hörfunksenders sollte nicht unbedingt ausgeklammert werden. Wenn also ein wirtschaftliches Arbeiten zunächst nur in Ballungsräumen möglich sein wird, so sollte das nicht dazu verführen, ausgesprochene Lokalprogramme anzusteuern, wie das von Seiten der Zeitungsverleger überlegt wird. Ein wirklich ansehnlicher Teil des Fernsehpublikums ist mit reinen Lokalprogrammen nicht $\mathrm{zu}$ erreichen.

Die Lösung kann nur ein Netzwerk-Programm mit sogenannten Fensterprogrammen bieten, das heißt: ein bundesweit ausgestrahltes Normalprogramm, dem Regionalbzw. Lokalsendungen einschließlich Werbung zeitlich vorgeschaltet werden können. Im Hinblick auf die technische Zukunftsentwicklung, insbesondere auf die kommen- 
den Möglichkeiten durch Fernsehsatelliten, wäre eine Entscheidung für das Lokalfernsehen unverständlich. Für die Gestaltung des Gesamtprogramms könnten aus.reichend vorhandene Kapazitäten freier Produktionen herangezogen werden. Es würde dem pluralen Charakter unserer Gesellschaft angemessen sein, wenn endlich der konsequente Versuch zur Gestaltung eines Kontrastprogramms gemacht würde.

\section{Baldige Klärungen erforderlich}

Der Zeitraum bis 1971 ist für die Strukturierung einer Fernsehanstalt neuen Typs relativ kurz. Wenn es nicht zu einem Parforceritt in letzter Stunde kommen soll, müßte unverzüglich mit den erforderlichen Vorklärungen und Vorbereitungen begonnen werden. Gewiß stellt sich eine Fülle von Problemen, doch ist keines unlösbar, wenn von Anfang an unvoreingenommene Fachleute herangezogen werden, zumal dic weltweite Entwicklung des Fernsehens eine Vielzahl von Erfahrungen anbietet, die genutzt werden können. Nicht nur Interessenbekundungen in Fülle, sondern auch Initiativen sehr konkreter Art wird es in Kürze geben. Absichten und Zielsetzungen werden jeweils sorgfältig zu prüfen sein. Es steht fest, daß die Zukunft des Fernsehens wieder völlig offen ist. Die Kirchen haben in diesem Zusammenhang die Fragen zu beantworten, ob sie auch weiterhin nur kulturkritisch tätig sein wollen, oder ob sie bereit und in der Lage sind, ihren hohen Verantwortungsanspruch in konstruktive und produktive Mitwirkung umzusetzen.

\section{S UM M A R Y}

As early as in 1971 television in the Federal Republic of Germany will have undergone some major changes. Technological development (channel VI) will allow to operate another national network, a "Fourth Program". A number of regional or local programs will be available. Legislative problems existing may soon find a reasonable solution. The churches already abandoned their critical attitude toward privately owned stations and are now seeking adequate means of constructive participation. They emphasize education in media usage and vigorous support for the production of quality programs. Kraemer indicates a profitable way by which the churches, the press and a group of cultural institutions could find some viable financial basis for a television association.

\section{R E S U MEN}

Ya para el año 1971 la televisión deja de estar limitada a las trasmisiónes hasta ahora existentes. $\mathrm{La}$ incorporación a un nuevo rango de frecuencia (12 Giga-Hertz / Band VI) ofrece los prerequisitos tecnicos, tanto para un cuarto programa difundido atraves de todo el territorio, como también para un gran número de programas regionales o locales. Los obstáculos legales aun presentes no son tampoco insuperables. Las dos iglesias han abandonado su reservada actitud ante las emisoras privadas y se muestran dispuestas a participar. A ellas se le presenta en esa nueva situación un doble tarea: intensificar la educación en el campo de las comunicaciónes de masas y también el fomento activo de un programa de alta calificación. Kraemer hace alución a una cooperación posible, entre las iglesias, la prensa y una comunidad de instituciónes culturales, cooperación que sería capacitada para realizar inversiones importantes. Un pequeño intervalo de tiempo nos separa del año 1971 y una vez más el futuro de la televisión se vuelve abrir. Cabe a las iglesias una actividad no solamente cultural, sino también la tarea de traducir la responsabilidad que ella se atribuye, en cooperación constructiva. 